\title{
Association between frequency of spicy food consumption and hypertension: a cross-sectional study in Zhejiang Province, China
}

Hao Wang ${ }^{1}$, Lingli Chen ${ }^{2}$, Dun Shen ${ }^{2}$, Yuan Cao ${ }^{2}$, Xiaoyi Zhang ${ }^{2}$, Kaixu Xie ${ }^{2}$, Chunmei Wang ${ }^{2}$, Shuiqing Zhu², Pei Pei ${ }^{3}$, Yu Guo ${ }^{3}$, Fiona Bragg ${ }^{4}$, Min Yu ${ }^{1 *}$, Zhengming Chen ${ }^{4}$ and Liming Li $i^{5,6}$

\begin{abstract}
Background: Hypertension is a known risk factor for multiple chronic diseases. Existing literature on the association between frequency of spicy food consumption and hypertension shows mixed findings.

Methods: The analyses are based on the Tongxiang baseline dataset of the China Kadoorie Biobank prospective study, including data from electronic questionnaires, physical measurements and blood sample collection. A total of 53,916 participants aged 30-79 years were included in the final analysis. Multivariable logistic regression was used to estimate the association of spicy food consumption with hypertension, and multiple linear regression was performed to explore the association of spicy food consumption with systolic and diastolic blood pressure.

Results: Of the 53,916 participants, 23,921 had prevalent hypertension. 12.3\% of participants reported consuming spicy food weekly. Among female participants, after adjusting for socio-demographic status, lifestyle factors, BMl, waist circumference, sleep duration and snoring, when compared with females who never consumed spicy food, the odds ratios $(95 \% \mathrm{Cl})$ for hypertension were $1.02(0.96-1.08), 0.90(0.79-1.01)$, and $0.88(0.78-0.99)$, respectively, for females who consumed spicy food less than once weekly, $1-2$ times weekly, and $\geq 3$ times weekly $\left(P_{\text {trend }}=0.04\right)$. The corresponding odds ratios for males were $1.02(0.95-1.09), 1.07(0.95-1.20)$, and 0.91 (0.81-1.01), respectively $\left(P_{\text {trend }}=0.39\right)$. Among current alcohol drinkers, compared to participants who never consumed spicy food, the odds ratio $(95 \% \mathrm{Cl})$ for hypertension among participants consuming spicy food daily was 0.98 (0.80-1.20). The corresponding figure for non-current drinkers was $0.72(0.62-0.84)$. The association was stronger among non-current alcohol drinkers than among current drinkers $\left(P_{\text {heterogeneity }}=0.02\right.$ ).
\end{abstract}

Conclusions: Frequency of spicy food consumption is inversely associated with hypertension in females, but not in males.

Keywords: Hypertension, Spicy food, Cross-sectional study

*Correspondence: myu@cdc.zj.cn

1 Department of NCDs Control and Prevention, Zhejiang Provincial Center

for Diseases Control and Prevention, \#3399 Binsheng Road, Binjiang District, Hangzhou, Zhejiang Province, China

Full list of author information is available at the end of the article

\section{Background}

Hypertension is one of the main causes of cardiovascular diseases [1, 2]. High systolic blood pressure is the leading risk factor for death in China, and was estimated to account for 2.54 million deaths in 2017, of which $95.7 \%$ were due to cardiovascular diseases [3]. 
Recently, a nationally representative survey, including 451,755 adults over 18 years of age from 31 provinces in mainland China, indicated that $23.2 \%(\approx 244.5$ million $)$ of Chinese adults had hypertension, and $41.3 \%(\approx 435.3$ million) had prehypertension [4]. Hence, hypertension remains a serious public health problem in China.

Worldwide, spices are an essential part of culinary cultures, with a long history of use for flavoring, coloring and preserving food, as well as for medicinal purposes $[5,6]$. Spices are recognized as one of the primary tastes in ancient Asia, especially in India and China [7, 8].

In past decades, the effects of spicy food consumption on chronic diseases and other conditions, including obesity, cancer, ischemic heart disease, cerebrovascular disease, fracture, dyslipidemia and impaired cognitive function, have been studied [9-15]. Some studies suggest lower disease risks associated with consumption of spicy food [11, 12, 14], while others suggest disease risks are higher $[9,10,15]$. The association between spicy food and hypertension has received considerable attention in recent years. However, the results from existing studies are similarly controversial [12, 16-18]. For example, while Ahuja and colleagues found that four weeks of regular chilli consumption had no obvious effects on blood pressure among healthy free-living individuals [16], Tingchao et al. reported that frequency of spicy food consumption was inversely associated with risk of hypertension in women [12]. Hence, the aim of this study was to examine the association of frequency of spicy food consumption with hypertension using data from the CKB study in Tongxiang, Zhejiang.

\section{Methods}

\section{Study population and design}

Detailed information about the CKB study design, survey methods and population has been described elsewhere [19-21]. The data utilized in the current study were obtained from Tongxiang, one of the 10 regions included in the CKB study. In brief, 57,704 participants aged 30-79 years were recruited and participated in the baseline survey between August 2004 and January 2008. The baseline survey consisted of a questionnaire, physical measurements and blood sample collection. All survey operations were conducted by trained, qualified staff using standardized procedures. For the current study, participants who had a history of cancer $(n=163)$, stroke $(\mathrm{n}=349)$, heart disease $(\mathrm{n}=464)$, physician-diagnosed diabetes $(n=1380)$, or baseline screen-detected diabetes $(n=1432)$ were excluded. After these exclusions, a total of 53,916 (22,573 men, 31,343 women) participants remained for inclusion in the final analyses.

\section{Outcome variable}

Blood pressure was measured in a seated position at least twice using a digital sphygmomanometer (Omron UA-779) in a quiet room with constant indoor temperature around $20{ }^{\circ} \mathrm{C}$. Two measurements were undertaken with a 5-min interval between measurements. If the difference between the first systolic blood pressure (SBP) and second SBP measurement was greater than $10 \mathrm{~mm}$ $\mathrm{Hg}$, a third measurement was conducted and average of the last two measurements was recorded and used for analyses [1]. Participants were considered to be hypertensive if they had a measured $\mathrm{SBP} \geq 140 \mathrm{~mm} \mathrm{Hg}$, a measured diastolic blood pressure (DBP) $\geq 90 \mathrm{~mm} \mathrm{Hg}$, or reported a prior history of doctor-diagnosed hypertension or use of antihypertensive medication.

\section{Assessment of exposure variable}

Frequency of spicy food consumption was assessed through the question "how often did you eat hot spicy food during the past month?". Answer options included: "Never or almost never" (i.e., non-consumers), "only occasionally" (i.e., less than once weekly), "1-2 days/ week", "3-5 days/week", and "daily or almost every day". In analyses, those who chose " $3-5$ days/week" or "daily or almost every day"' were combined into one group (i.e., $\geq 3$ days/week). The reproducibility of frequency of spicy food consumption was tested twice with a median interval of 1.4 years. Spearman's coefficient for the correlation was 0.71 , indicating that spicy food consumption was reported consistently [11].

\section{Assessment of covariates}

An interviewer-administered electronic questionnaire included socio-demographic characteristics (age, sex, education level, marital status, and household income), lifestyle factors (cigarette smoking, alcohol drinking, physical activity, fresh fruit intake, meat intake, sleep duration and snoring), personal medical history (cancer, stroke, diabetes, and heart attack), family history of hypertension, and, among women, menopause status.

Cigarette smoking and alcohol drinking were categorized into four groups according to participants' responses: (1) non-smokers (or non-drinkers); (2) former smokers (or former drinkers); (3) occasional smokers (or occasional drinkers); and (4) current smokers (or current drinkers) [22, 23]. Total physical activity was converted into metabolic equivalent of task hours per day (MET-hours/day) based on transportation, occupation, housework, and non-sedentary recreation as described in previous studies [24, 25].

Physical measurements included height, weight, and waist circumference (WC), measured using calibrated 
instruments by trained health workers. Standing height was measured to the nearest $0.1 \mathrm{~cm}$ with a stadiometer. Weight was measured to the nearest $0.1 \mathrm{~kg}$ with a body composition analyzer. Body mass index (BMI) was calculated as weight in kilograms divided by the square of standing height in meters, and obesity was defined as $\mathrm{BMI} \geq 25.0 \mathrm{~kg} / \mathrm{m}^{2}$ [26]. WC was measured to the nearest $0.1 \mathrm{~cm}$ with a non-stretchable tape measure at the midpoint between the lowest rib and the iliac crest. Excessive WC was defined as $\geq 85 \mathrm{~cm}$ for males, and $\geq 80 \mathrm{~cm}$ for females [27]. A non-fasting venous blood sample was collected. Immediate on-site testing of plasma glucose level was undertaken.

\section{Statistical analysis}

Statistical analyses were performed using SAS 9.4 (SAS Institute Inc., Cary, NC, USA). Descriptive statistics were presented as mean \pm SD or percentages for continuous or categorical variables, respectively. To examine the association between frequency of spicy food consumption and risk of hypertension, univariate and multivariable logistic regression analyses were applied. Participants who never consumed spicy food were considered as the reference group. Potential confounding factors, including sociodemographic status and lifestyle factors were adjusted for in different models. In model 1 , odds ratios were adjusted for age (continuous) and sex. Model 2 included additional adjustment for education level (no formal school, primary school, middle school, and high school or above) and household income ( $\leq 19,999$ yuan, 20,000-34,999 yuan, $\geq 35,000$ yuan). Model 3 included additional adjustment for cigarette smoking (never, occasional, former, and current), alcohol drinking (never, occasional, former, and current), physical activity (continuous), meat consumption (daily and non-daily), fruit consumption (daily and non-daily), BMI (continuous), WC (continuous), snoring (never, occasional, and habitual) and sleep duration (continuous). Multiple linear regression analyses were further performed to explore the associations of frequency of spicy food consumption with systolic blood pressure and diastolic blood pressure. Stratified analyses were conducted to detect whether the associations of daily spicy food consumption with prevalent hypertension differed according to age (30-49 y or 50-79 y), education level (illiterate or primary, and above), household income $(<35,000$ yuan or $\geq 35,000$ yuan), physical activity ( $<30 \mathrm{MET}-\mathrm{h} / \mathrm{d}$ or $\geq 30 \mathrm{MET}-\mathrm{h} / \mathrm{d})$, smoking status (current smokers or non-current smokers), alcohol status (current drinkers or non-current drinkers), meat consumption (daily or non-daily), fruit consumption (daily or non-daily), BMI $\left(<25 \mathrm{~kg} / \mathrm{m}^{2}\right.$ or $\left.\geq 25 \mathrm{~kg} / \mathrm{m}^{2}\right)$, WC (normal or excessive), sleep duration $(<7.6 \mathrm{~h} / \mathrm{d}$ or $\geq 7.6 \mathrm{~h} / \mathrm{d})$, or menopause status (menopausal or non-menopausal). In sensitivity analyses, 1128 participants with self-reported physician-diagnosed peptic ulcer disease were excluded from the analyses. All statistical significance was set at $P=0.05$.

\section{Results}

\section{Characteristics of participants}

Of the 53,916 participants, 23,921 had prevalent hypertension. The prevalence of hypertension among participants who consumed spicy food never, less than once per week, 1-2 times weekly, and $\geq 3$ times weekly were $64.0 \%, 23.7 \%, 5.8 \%$ and $6.5 \%$, respectively.

Compared with non-consumers, participants who consumed spicy food frequently were more likely to be younger, male, well-educated, current smokers, current drinkers, habitual snorers, physically inactive, to consume meat and fruit frequently, to have higher BMI and WC, and to report longer sleep duration. No significant difference was found in household income $(P=0.51)$ or in menopause status $(P=0.65)$ according to frequency of spicy food consumption (Table 1).

\section{Association of frequency of spicy food consumption with hypertension}

In univariate analysis, compared to non-consumers, odds ratios $(95 \% \mathrm{CI})$ for hypertension among participants who consumed spicy food less than once per week, 1-2 times per week, and $\geq 3$ times per week were 0.89 (0.86-0.93), 0.77 (0.71-0.83), and $0.74(0.69-0.79)$, respectively.

After adjustment for age, sex, education level, and household income, participants who consumed spicy food less than once per week, 1-2 times per week, and $\geq 3$ times per week had odds ratios $(95 \% \mathrm{CI})$ for hypertension of 1.05 (1.01-1.10), 1.03 (0.95-1.11), and 0.97 (0.90-1.04), respectively, when compared with non-consumers. After further adjustment for cigarette smoking, alcohol drinking, physical activity, meat intake, fruit intake, BMI, WC, self-reported snoring, and sleep duration, the associations were attenuated, with corresponding odds ratios (95\% CI) of 1.01 (0.97-1.06), 0.98 (0.91-1.07), and $0.88(0.82-0.96)$, respectively. When the association was explored separately among women, the corresponding odds ratios $(95 \% \mathrm{CI})$ were $1.02(0.96-$ $1.08), 0.90(0.79-1.01)$, and $0.88(0.78-0.99)$, respectively $\left(P_{\text {trend }}=0.04\right)$. However, such an inverse association was not found among males $\left(P_{\text {trend }}=0.39\right)($ Table 2$)$.

\section{Association of frequency of spicy food consumption and blood pressure}

After adjusting for potential confounders in model 3 (age, sex, socio-demographic and lifestyle factors, adiposity, snoring and sleep duration), compared with non-consumers, the adjusted $\beta$ coefficients (95\% CI) for systolic 
Table 1 Baseline characteristics of participants according to frequency of spicy food consumption

\begin{tabular}{|c|c|c|c|c|c|c|}
\hline \multirow[t]{2}{*}{ Characteristics } & \multirow[t]{2}{*}{ Overall $(\mathrm{N}=53,916)$} & \multicolumn{4}{|c|}{ Frequency of spicy food consumption } & \multirow[t]{2}{*}{$P_{\text {trend }}$} \\
\hline & & Never $(\mathrm{N}=34,513)$ & $\begin{array}{l}<1 \text { times/week } \\
(\mathrm{N}=12,781)\end{array}$ & $\begin{array}{l}1-2 \text { times/ } \\
\text { week } \\
(\mathrm{N}=3109)\end{array}$ & $\begin{array}{l}\geq 3 \text { times/week } \\
(\mathrm{N}=3513)\end{array}$ & \\
\hline Mean age $\left(\right.$ years) ${ }^{a}$ & $52.5 \pm 9.9$ & $53.8 \pm 9.9$ & $50.8 \pm 9.4$ & $48.6 \pm 8.8$ & $48.5 \pm 9.0$ & $<0.0001$ \\
\hline Females $(\%)^{b}$ & 58.1 & 62.2 & 53.2 & 47.3 & 43.4 & $<0.0001$ \\
\hline No formal education (\%) & 43.5 & 44.5 & 41.1 & 39.5 & 41.9 & $<0.0001$ \\
\hline Income $\geq 35,000$ (yuan) (\%) & 37.8 & 36.2 & 43.8 & 33.1 & 34.6 & 0.51 \\
\hline Current smokers (\%) & 28.0 & 26.9 & 29.1 & 29.7 & 31.3 & $<0.0001$ \\
\hline Current drinkers (\%) & 17.1 & 14.1 & 20.0 & 22.8 & 26.9 & $<0.0001$ \\
\hline Physical activities (MET-h/d) & $30.6 \pm 15.3$ & $30.6 \pm 15.2$ & $31.0 \pm 15.2$ & $30.7 \pm 15.0$ & $30.2 \pm 15.9$ & 0.0024 \\
\hline Consuming meat daily (\%) & 15.2 & 13.2 & 16.5 & 20.4 & 24.5 & $<0.0001$ \\
\hline Consuming fruit daily (\%) & 6.7 & 5.6 & 6.7 & 11.6 & 14.6 & $<0.0001$ \\
\hline $\mathrm{BMI}\left(\mathrm{kg} / \mathrm{m}^{2}\right)$ & $22.9 \pm 3.1$ & $22.8 \pm 3.1$ & $23.0 \pm 3.1$ & $23.0 \pm 3.0$ & $23.3 \pm 3.1$ & $<0.0001$ \\
\hline WC $(\mathrm{cm})$ & $76.5 \pm 9.1$ & $76.3 \pm 9.0$ & $76.9 \pm 9.0$ & $76.6 \pm 9.1$ & $77.1 \pm 9.3$ & $<0.0001$ \\
\hline Habitual snoring (\%) & 24.3 & 23.4 & 26.0 & 25.3 & 26.7 & $<0.0001$ \\
\hline Sleep duration (hours) & $7.6 \pm 1.2$ & $7.6 \pm 1.2$ & $7.6 \pm 1.1$ & $7.8 \pm 1.1$ & $7.7 \pm 1.2$ & $<0.0001$ \\
\hline Women with menopause $(\%)^{\mathrm{b}, \mathrm{c}}$ & 53.9 & 54.0 & 53.0 & 52.5 & 52.6 & 0.65 \\
\hline
\end{tabular}

Data were adjusted for age and sex unless specified

MET metabolic equivalent of task, BMI body mass index, WC waist circumference

${ }^{\text {a }}$ Adjusted for sex

${ }^{\mathrm{b}}$ Adjusted for age

c Only in women

blood pressure associated with consumption of spicy food $<1$ times/week, $1-2$ times/week, and $\geq 3$ times/week were $0.31(-0.08,0.71),-0.41(-1.13,0.31)$, and -0.96 $(-1.64,-0.27)$, respectively $\left(P_{\text {trend }}<0.05\right)$. When the association was examined separately among women, the corresponding $\beta$ coefficients (95\% CI) were $0.46(-0.08$, $0.99),-0.37(-1.39,0.64)$, and $-1.06(-2.06,-0.07)$, respectively $\left(P_{\text {trend }}<0.05\right)$ i.e., there was an inverse association between frequency of spicy food consumption and systolic blood pressure among females. However, there was no statistically significant trend observed among males $\left(P_{\text {trend }}=0.24\right)$ (Table 3).

Similar to the association with systolic blood pressure, frequency of spicy food consumption was negatively related to diastolic blood pressure among females $\left(P_{\text {trend }}<0.05\right)$, but no significant association was observed among males $\left(P_{\text {trend }}=0.38\right)$ (Table 4$)$.

\section{Subgroup analyses}

In subgroup analyses, the strength of the association between frequency of spicy food consumption and hypertension was largely consistent across subgroups defined by age, education level, household income, physical activity, cigarette smoking, meat consumption, fruit consumption, BMI, WC, sleep duration, and menopause status $\left(P_{\text {heterogeneity }}>0.05\right)$. However, there was a significantly stronger association among non-current alcohol drinkers $(\mathrm{OR}=0.72,95 \% \mathrm{CI}, 0.62-0.84)$ than among current alcohol drinkers $(\mathrm{OR}=0.98,95 \% \mathrm{CI}$, $0.80-1.20)\left(P_{\text {heterogeneity }}=0.02\right)$ (Table 5).

\section{Sensitivity analyses}

In sensitivity analyses, after exclusion of 1128 participants with self-reported physician-diagnosed peptic ulcer disease, there was no marked change in the association of frequency of spicy food consumption with hypertension. Among female participants, compared to non-consumers, odds ratios $(95 \% \mathrm{CI})$ for hypertension among those who consumed spicy food less than once per week, $1-2$ times per week, and $\geq 3$ times per week were $1.02(0.96-1.09), 0.89(0.79-1.01)$, and 0.88 (0.78$0.99)$, respectively $\left(P_{\text {trend }}=0.04\right)$. The corresponding figures for males were 1.01 (0.95-1.09), 1.08 (0.96$1.21)$, and $0.92(0.83-1.03)$, respectively $\left(P_{\text {trend }}=0.39\right)$ (Additional file 1: Table S1).

\section{Discussion}

This large cross-sectional study explored the association of spicy food consumption with prevalent hypertension. Frequent spicy food consumption was inversely associated with hypertension in females. However, such an inverse association was not found in males. 
Table 2 Unadjusted and adjusted odds ratios for hypertension associated with frequency of spicy food consumption among adults in Zhejiang

\begin{tabular}{|c|c|c|c|c|c|}
\hline \multirow{2}{*}{$\begin{array}{l}\text { Frequency of spicy food } \\
\text { intake }\end{array}$} & \multirow[t]{2}{*}{ N. participants } & \multirow[t]{2}{*}{ Univariate } & \multicolumn{3}{|l|}{ Multivariable } \\
\hline & & & Model 1 & Model 2 & Model 3 \\
\hline \multicolumn{6}{|l|}{ Total } \\
\hline Never & 34,513 & 1.00 & 1.00 & 1.00 & 1.00 \\
\hline$<1$ times/week & 12,781 & $0.89(0.86-0.93)$ & $1.06(1.01-1.10)$ & $1.05(1.01-1.10)$ & $1.01(0.97-1.06)$ \\
\hline 1-2 times/week & 3109 & $0.77(0.71-0.83)$ & $1.02(0.94-1.10)$ & $1.03(0.95-1.11)$ & $0.98(0.91-1.07)$ \\
\hline$\geq 3$ times/week & 3513 & $0.74(0.69-0.79)$ & $0.96(0.89-1.04)$ & $0.97(0.90-1.04)$ & $0.88(0.82-0.96)$ \\
\hline$P_{\text {trend }}$ & & $<0.0001$ & 0.86 & 0.74 & 0.02 \\
\hline \multicolumn{6}{|l|}{ Males $^{a}$} \\
\hline Never & 13,211 & 1.00 & 1.00 & 1.00 & 1.00 \\
\hline$<1$ times/week & 5900 & $0.92(0.87-0.98)$ & $1.10(1.03-1.17)$ & $1.08(1.01-1.15)$ & $1.02(0.95-1.09)$ \\
\hline 1-2 times/week & 1581 & $0.89(0.80-0.99)$ & $1.17(1.05-1.30)$ & $1.17(1.05-1.31)$ & $1.07(0.95-1.20)$ \\
\hline$\geq 3$ times/week & 1881 & $0.83(0.75-0.92)$ & $1.05(0.95-1.16)$ & $1.05(0.95-1.16)$ & $0.91(0.81-1.01)$ \\
\hline$P_{\text {trend }}$ & & $<0.0001$ & 0.02 & 0.02 & 0.39 \\
\hline \multicolumn{6}{|l|}{ Females $^{a}$} \\
\hline Never & 21,302 & 1.00 & 1.00 & 1.00 & 1.00 \\
\hline$<1$ times/week & 6881 & $0.85(0.80-0.90)$ & $1.03(0.97-1.09)$ & $1.03(0.97-1.09)$ & $1.02(0.96-1.08)$ \\
\hline 1-2 times/week & 1528 & $0.62(0.56-0.69)$ & $0.87(0.78-0.98)$ & $0.89(0.79-0.99)$ & $0.90(0.79-1.01)$ \\
\hline$\geq 3$ times/week & 1632 & $0.60(0.54-0.67)$ & $0.88(0.79-0.98)$ & $0.90(0.80-1.00)$ & $0.88(0.78-0.99)$ \\
\hline$P_{\text {trend }}$ & & $<0.0001$ & 0.02 & 0.06 & 0.04 \\
\hline
\end{tabular}

In model 1, odds ratios were adjusted for age (continuous) and sex. Model 2 included additional adjustment for education level (no formal school, primary school, middle school, and high school or above), household income ( $\leq 19,999$ yuan, 20,000-34,999 yuan, $\geq 35,000$ yuan), Model 3 included additional adjustment for cigarettes consumption (never, occasional, former, and current), alcohol consumption (never, occasional, former, and current), physical activity (continuous), meat consumption (daily and non-daily), fruit consumption (daily and non-daily), BMI (continuous), WC (continuous), snoring (never, occasional, and habitual), sleep duration (continuous)

${ }^{\text {a }}$ Without adjustment for sex

\section{Frequency of spicy food consumption}

A previously published paper from the CKB study indicated that $99.7 \%$ of participants in Hunan consumed spicy food weekly, while only $8.8 \%$ of participants in Haikou consumed spicy food weekly [9]. In the present study, the prevalence of weekly spicy food consumption was $12.3 \%$, much lower than in the CKB population as a whole, in which $42.5 \%$ of participants consumed spicy food weekly [9]. This discrepancy reflects geographic distribution of Chinese residents' preferences for spicy food. Zhejiang is located in the east of China, and compared with residents living in most other provinces, residents in Zhejiang have a preference for a more bland diet rather than heavy tastes.

Consistent with previous studies [12, 28], participants who more frequently consumed spicy food were more likely to be young, male, current smokers, alcohol drinkers, and to more frequently eat meat and fruit. In contrast with an earlier study, indicating that individuals with high levels of spicy food consumption had lower BMI levels when compared with non-consumers [17], the present study documented that individuals who consumed spicy food more frequently seemed to have higher BMI and WC than non-consumers. This may reflect investigation of crude BMI values, without adjustment for age. Sun et al. found that spicy food consumption was positively associated with adiposity, including both general and abdominal obesity [9], similar with the current study. This positive association may reflect increased palatability of meals including spicy food [7].

\section{Relationship of frequency of spicy food consumption with hypertension}

Harada et al. found that SBP and DBP were significantly lower among hypertensive volunteers after administration of a mixture of capsaicin and isoflavone for 5 months, but not among normotensive volunteers [18]. In a randomized cross-over dietary intervention study from Australia, 36 individuals (22 women and 14 men) consumed a chilli diet ( $30 \mathrm{~g}$ chilli per day) and a bland diet (chilli-free) for 4 weeks each [16]. This study concluded that four weeks of regular chilli consumption had no obvious beneficial or harmful effects on SBP or DBP, which was inconsistent with the present study. However, a prospective cohort involving 13,670 Chinese adults [17], with a median follow-up of 9.0 -years, demonstrated 
Table 3 Unadjusted and adjusted $\beta$ coefficients for systolic blood pressure associated with frequency of spicy food consumption among adults in Zhejiang

\begin{tabular}{|c|c|c|c|c|c|}
\hline & \multicolumn{4}{|c|}{ Frequency of spicy food consumption } & \multirow[t]{2}{*}{$P_{\text {trend }}$} \\
\hline & Never & $<1$ times/week & 1-2 times/week & $\geq 3$ times/week & \\
\hline N. participants (total) & 34,513 & 12,781 & 3109 & 3513 & \\
\hline $\mathrm{SBP}, \mathrm{mmHg}$ & $135.9 \pm 21.4$ & $134.8 \pm 20.8$ & $132.6 \pm 20.2$ & $132.6 \pm 20.7$ & \\
\hline Unadjusted $\beta$ (95\% Cl) & Ref & $-1.14(-1.57,-0.71)$ & $-3.39(-4.16,-2.61)$ & $-3.36(-4.09,-2.62)$ & $<0.0001$ \\
\hline Model 1 adjusted $\beta(95 \% \mathrm{Cl})$ & Ref & $0.73(0.32,1.14)$ & $-0.20(-0.94,0.55)$ & $-0.30(-1.00,0.41)$ & 0.88 \\
\hline Model 2 adjusted $\beta(95 \% \mathrm{Cl})$ & Ref & $0.74(0.32,1.15)$ & $-0.06(-0.81,0.69)$ & $-0.21(-0.92,0.49)$ & 0.63 \\
\hline Model 3 adjusted $\beta(95 \% \mathrm{Cl})$ & Ref & $0.31(-0.08,0.71)$ & $-0.41(-1.13,0.31)$ & $-0.96(-1.64,-0.27)$ & 0.03 \\
\hline N. participants (males) $)^{a}$ & 13,211 & 5900 & 1581 & 1881 & \\
\hline $\mathrm{SBP}, \mathrm{mmHg}$ & $137.0 \pm 20.8$ & $136.3 \pm 19.9$ & $134.8 \pm 19.7$ & $135.5 \pm 20.4$ & \\
\hline Unadjusted $\beta$ (95\% Cl) & Ref & $-0.74(-1.37,-0.11)$ & $-2.24(-3.31,-1.17)$ & $-1.55(-2.54,-0.56)$ & $<0.0001$ \\
\hline Model 1 adjusted $\beta$ (95\% Cl) & Ref & $1.02(0.40,1.63)$ & $0.52(-0.52,1.56)$ & $0.84(-0.12,1.80)$ & 0.01 \\
\hline Model 2 adjusted $\beta$ (95\% Cl) & Ref & $0.94(0.32,1.55)$ & $0.61(-0.43,1.65)$ & $0.87(-0.09,1.84)$ & 0.01 \\
\hline Model 3 adjusted $\beta(95 \% \mathrm{Cl})$ & Ref & $0.19(-0.40,0.79)$ & $-0.43(-1.44,0.57)$ & $-0.60(-1.54,0.34)$ & 0.24 \\
\hline N. participants (females) $)^{\mathrm{a}}$ & 21,302 & 6881 & 1528 & 1632 & \\
\hline $\mathrm{SBP}, \mathrm{mmHg}$ & $135.3 \pm 21.8$ & $133.5 \pm 21.5$ & $130.3 \pm 20.4$ & $129.3 \pm 20.6$ & \\
\hline Unadjusted $\beta$ (95\% Cl) & Ref & $-1.74(-2.33,-1.15)$ & $-5.01(-6.13,-3.89)$ & $-6.01(-7.09,-4.92)$ & $<0.0001$ \\
\hline Model 1 adjusted $\beta$ (95\% Cl) & Ref & $0.52(-0.04,1.08)$ & $-0.92(-1.98,0.14)$ & $-1.44(-2.47,-0.41)$ & 0.03 \\
\hline Model 2 adjusted $\beta(95 \% \mathrm{Cl})$ & Ref & $0.59(0.04,1.15)$ & $-0.69(-1.75,0.37)$ & $-1.23(-2.27,-0.20)$ & 0.12 \\
\hline Model 3 adjusted $\beta(95 \% \mathrm{Cl})$ & Ref & $0.46(-0.08,0.99)$ & $-0.37(-1.39,0.64)$ & $-1.06(-2.06,-0.07)$ & 0.03 \\
\hline
\end{tabular}

In model 1, odds ratios were adjusted for age (continuous) and sex. Model 2 included additional adjustment for education level (no formal school, primary school, middle school and high school or above), household income ( $\leq 19,999$ yuan, 20,000-34,999 yuan, $\geq 35,000$ yuan), Model 3 included additional adjustment for cigarettes consumption (never, occasional, former, and current), alcohol consumption (never, occasional, former, and current), physical activity (continuous), meat consumption (daily and non-daily), fruit consumption (daily and non-daily), BMI (continuous), WC (continuous), snoring (never, occasional, and habitual snoring), sleep duration (continuous)

${ }^{\text {a }}$ Without adjustment for sex

that, after adjustment for age, gender, energy, sodium and fat intake, smoking, alcohol, and physical activity, when compared with non-consumers, the HRs (95\% CI) for incident hypertension among participants who consumed chilli in quantities of $1-20 \mathrm{~g} /$ day, $20.1-50 \mathrm{~g} /$ day and $\geq 50.1 \mathrm{~g} /$ day were $0.80(0.73-0.88), 0.81(0.73-0.89)$ and $0.65(0.57-0.75)$, respectively, consistent with the current study.

The 2009 China Health and Nutrition Survey (CHNS), including 9273 adults aged $\geq 18$ years old, was conducted in nine geographically diverse provinces. Findings from the CHNS indicated that, compared with females who did not eat spicy food, the adjusted odds ratios (95\% CI) of hypertension for women who consumed spicy food 1-2 times/week, 3-4 times/week, and $\geq 5$ times/ week were $0.92(0.78-1.09), 0.91(0.73-1.13)$, and 0.74 (0.57-0.96), respectively, but this inverse association was not found in men [12]. This sex disparity in the association between frequency of spicy food consumption and hypertension was compatible with the current study.

Two large prospective population studies conducted in China and Italy illustrated that spicy food intake was associated with a lower risk of death due to cardiovascular diseases [11, 14]. High blood pressure is a well-known risk factor for cardiovascular diseases [1, 2], and the inverse association between spicy food consumption and hypertension found in the current study suggests the apparent protective effect of spicy food on cardiovascular disease mortality may be mediated via lowering of blood pressure.

In subgroup analyses, significant differences in the association of spicy food consumption with hypertension were observed across strata of alcohol consumption, with a stronger inverse association among non-current drinkers than current drinkers. Intriguingly, prospective analyses based on 0.48 million CKB participants showed that the inverse association of spicy food consumption with all-cause mortality was stronger in participants who did not drink alcohol than among those who did drink alcohol [11], which is in line with the current study.

Although mechanisms underlying the potential beneficial effect of spicy food consumption on hypertension have not yet been fully elucidated, several hypotheses have been proposed. First, capsaicin, as the major pungent element in red pepper, is a neurotoxic agent, and could activate transient receptor potential 
Table 4 Unadjusted and adjusted $\beta$ coefficients for diastolic blood pressure associated with frequency of spicy food consumption among adults in Zhejiang

\begin{tabular}{|c|c|c|c|c|c|}
\hline & \multicolumn{4}{|c|}{ Frequency of spicy food consumption } & \multirow[t]{2}{*}{$P_{\text {trend }}$} \\
\hline & Never & $<1$ times/week & 1-2 times/week & $\geq 3$ times/week & \\
\hline N. participants (total) & 34,513 & 12,781 & 3109 & 3513 & \\
\hline $\mathrm{DBP}, \mathrm{mmHg}$ & $80.3 \pm 10.7$ & $80.6 \pm 10.8$ & $80.3 \pm 10.7$ & $80.5 \pm 11.1$ & \\
\hline Unadjusted $\beta(95 \% \mathrm{Cl})$ & Ref & $0.30(0.09,0.52)$ & $0.06(-0.34,0.45)$ & $0.20(-0.17,0.57)$ & 0.08 \\
\hline Model 1 adjusted $\beta(95 \% \mathrm{Cl})$ & Ref & $0.21(-0.01,0.43)$ & $-0.09(-0.48,0.31)$ & $-0.01(-0.38,0.36)$ & 0.75 \\
\hline Model 2 adjusted $\beta(95 \% \mathrm{Cl})$ & Ref & $0.18(-0.03,0.40)$ & $-0.02(-0.41,0.38)$ & $0.04(-0.33,0.41)$ & 0.55 \\
\hline Model 3 adjusted $\beta(95 \% \mathrm{Cl})$ & Ref & $-0.04(-0.26,0.17)$ & $-0.23(-0.61,0.15)$ & $-0.39(-0.75,-0.02)$ & 0.03 \\
\hline N. participants (males) ${ }^{a}$ & 13,211 & 5900 & 1581 & 1881 & \\
\hline $\mathrm{DBP}, \mathrm{mmHg}$ & $81.5 \pm 11.0$ & $82.0 \pm 11.0$ & $81.8 \pm 11.0$ & $82.1 \pm 11.5$ & \\
\hline Unadjusted $\beta$ (95\% Cl) & Ref & $0.45(0.11,0.79)$ & $0.30(-0.27,0.87)$ & $0.65(0.11,1.18)$ & 0.004 \\
\hline Model 1 adjusted $\beta(95 \% \mathrm{Cl})$ & Ref & $0.46(0.11,0.80)$ & $0.31(-0.27,0.89)$ & $0.66(0.12,1.19)$ & 0.004 \\
\hline Model 2 adjusted $\beta(95 \% \mathrm{Cl})$ & Ref & $0.36(0.02,0.71)$ & $0.36(-0.22,0.94)$ & $0.68(0.14,1.22)$ & 0.004 \\
\hline Model 3 adjusted $\beta(95 \% \mathrm{Cl})$ & Ref & $-0.07(-0.40,0.27)$ & $-0.24(-0.80,0.32)$ & $-0.16(-0.68,0.36)$ & 0.38 \\
\hline N. participants (females) $)^{\mathrm{a}}$ & 21,302 & 6881 & 1528 & 1632 & \\
\hline $\mathrm{DBP}, \mathrm{mmHg}$ & $79.5 \pm 10.4$ & $79.4 \pm 10.4$ & $78.8 \pm 10.3$ & $78.6 \pm 10.3$ & \\
\hline Unadjusted $\beta$ (95\% Cl) & Ref & $-0.11(-0.40,0.17)$ & $-0.70(-1.24,-0.16)$ & $-0.97(-1.49,-0.44)$ & $<0.0001$ \\
\hline Model 1 adjusted $\beta(95 \% \mathrm{Cl})$ & Ref & $0.02(-0.26,0.31)$ & $-0.45(-0.99,0.09)$ & $-0.69(-1.22,-0.16)$ & 0.01 \\
\hline Model 2 adjusted $\beta(95 \% \mathrm{Cl})$ & Ref & $0.05(-0.23,0.34)$ & $-0.35(-0.89,0.19)$ & $-0.59(-1.12,-0.06)$ & 0.04 \\
\hline Model 3 adjusted $\beta$ (95\% Cl) & Ref & $-0.03(-0.30,0.25)$ & $-0.25(-0.78,0.28)$ & $-0.57(-1.08,-0.05)$ & 0.04 \\
\hline
\end{tabular}

In model 1, $\beta$ coefficients were adjusted for age (continuous) and sex. Model 2 included additional adjustment for education level (no formal school, primary school, middle school and high school or above), household income ( $\leq 19,999$ yuan, 20,000-34,999 yuan, $\geq 35,000$ yuan), Model 3 included additional adjustment for cigarettes consumption (never, occasional, former, and current), alcohol consumption (never, occasional, former, and current), physical activity (continuous), meat consumption (daily and non-daily), fruit consumption (daily and non-daily), BMI (continuous), WC (continuous), snoring (never, occasional, and habitual snoring), sleep duration (continuous)

${ }^{a}$ Without adjustment for sex

vanilloid type-1 (TRPV1), in turn improving endothelium-dependent vasorelaxation and lowering blood pressure [29]. Second, activation of TRPV1 could reduce vascular lipid accumulation and attenuate atherosclerosis [30]. Third, activation of TRPV1 may prevent adipogenesis and obesity [31]. Lastly, enjoyment of spicy food may significantly reduce individual salt preference, daily salt intake, and blood pressure by modifying the neural processing of salty taste in the brain [32].

The findings of the current study are of potential clinical and public health importance. Firstly, spicy food might be a valuable dietary intervention for prevention of hypertension in both healthy populations and high-risk groups, especially in regions with typically low consumption of spicy food, such as Zhejiang. Secondly, spicy food should not be considered a dietary taboo for patients with hypertension. However, further evidence from prospective and randomized studies will be important in establishing this potential clinical relevance.

\section{Strengths and limitations}

The strengths of this study include a large sample size, use of standardized data collection procedures, and strict control for established and potential risk factors for hypertension. Several limitations merit mention, however. First, the cross-sectional design restricts establishment of the temporal relationship of spicy food consumption with hypertension. Second, assessment of spicy food consumption in the current study was self-reported and subject to measurement error. Third, although multiple established and potential risk factors for hypertension were adjusted for in different models, residual confounding by other unmeasured or unknown biological and social factors is still possible.

\section{Conclusions}

In conclusion, the current study shows that frequency of spicy food consumption is inversely associated with hypertension in females, but not in males. 
Table 5 Adjusted odds ratios for hypertension associated with consuming spicy food daily versus never according to participant characteristics

\begin{tabular}{|c|c|c|c|}
\hline & N. hypertension & ORs $(95 \% \mathrm{Cl})$ & $P_{\text {heterogeneity }}$ \\
\hline Age group (years) & & & 0.67 \\
\hline $30-49$ & 4139 & $0.76(0.64-0.90)$ & \\
\hline $50-79$ & 12,222 & $0.72(0.61-0.86)$ & \\
\hline Education level & & & 0.51 \\
\hline No formal education & 8739 & $0.86(0.70-1.04)$ & \\
\hline Primary or above & 7622 & $0.79(0.67-0.92)$ & \\
\hline Household income & & & 0.69 \\
\hline$<35,000$ (yuan) & 10,602 & $0.80(0.69-0.93)$ & \\
\hline$\geq 35,000$ (yuan) & 5759 & $0.84(0.68-1.04)$ & \\
\hline $\begin{array}{l}\text { Physical activity (MET- } \\
\text { h/d) }\end{array}$ & & & 0.06 \\
\hline$<30$ & 9344 & $0.72(0.61-0.86)$ & \\
\hline$\geq 30$ & 7017 & $0.91(0.77-1.08)$ & \\
\hline Smoking status & & & 0.14 \\
\hline Current smokers & 4059 & $0.90(0.75-1.08)$ & \\
\hline $\begin{array}{l}\text { Non-current smok- } \\
\text { ers }\end{array}$ & 12,302 & $0.75(0.63-0.88)$ & \\
\hline Alcohol status & & & 0.02 \\
\hline Current drinkers & 2654 & $0.98(0.80-1.20)$ & \\
\hline $\begin{array}{l}\text { Non-current drink- } \\
\text { ers }\end{array}$ & 13,707 & $0.72(0.62-0.84)$ & \\
\hline Meat consumption & & & 0.41 \\
\hline Daily & 2006 & $0.75(0.60-0.94)$ & \\
\hline Non-daily & 14,355 & $0.84(0.73-0.97)$ & \\
\hline Fruit consumption & & & 0.59 \\
\hline Daily & 795 & $0.88(0.63-1.22)$ & \\
\hline Non-daily & 15,566 & $0.80(0.70-0.91)$ & \\
\hline BMI $\left(\mathrm{kg} / \mathrm{m}^{2}\right)$ & & & 0.34 \\
\hline$<25$ & 11,260 & $0.86(0.74-0.99)$ & \\
\hline$\geq 25$ & 5101 & $0.76(0.61-0.94)$ & \\
\hline WC & & & 0.97 \\
\hline Normal & 7103 & $0.81(0.67-0.97)$ & \\
\hline Excessive & 9258 & $0.81(0.69-0.95)$ & \\
\hline $\begin{array}{l}\text { Sleep duration } \\
\text { (hours/day) }\end{array}$ & & & 0.29 \\
\hline$<7.6$ & 7377 & $0.75(0.61-0.91)$ & \\
\hline$\geq 7.6$ & 8984 & $0.85(0.73-0.99)$ & \\
\hline Menopause status & & & 0.94 \\
\hline Menopausal & 6841 & $0.80(0.59-1.08)$ & \\
\hline Non-menopausal & 2777 & $0.79(0.61-1.01)$ & \\
\hline
\end{tabular}

Odds ratios were adjusted for age (continuous) and sex, education level (no formal school, primary school, middle school and high school or above), household income ( $\leq 19,999$ yuan, 20,000-34 999 yuan, $\geq 35,000$ yuan), cigarettes consumption (never, occasional, former, and current), alcohol consumption (never, occasional, former, and current), physical activity (continuous), meat consumption (daily and non-daily), fruit consumption (daily and non-daily), BMI (continuous), WC (continuous), snoring (never, occasional, and habitual), sleep duration (continuous)

ORs odds ratios, $\mathrm{Cl}$ confidence intervals, $\mathrm{BMI}$ body mass index, WC waist circumference

\section{Abbreviations}

CKB: China Kadoorie Biobank; SBP: Systolic blood pressure; DBP: Diastolic blood pressure; MET: Metabolic equivalent tasks; BMI: Body mass index; WC: Waist circumference; ORs: Odds ratios; Cl: Confidence interval; HRs: Hazard ratios; CHNS: China health and nutrition survey; TRPV1: Transient receptor potential vanilloid type-1.

\section{Supplementary Information}

The online version contains supplementary material available at https://doi. org/10.1186/s12986-021-00588-7.

Additional file 1: Table S1. Unadjusted and adjusted odds ratios for hypertension associated with frequency of spicy food consumption among adults without peptic ulcer disease in Zhejiang.

\section{Acknowledgements}

We thank Chinese Center for Disease Control and Prevention, Chinese Ministry of Health, National Health and Family Planning Commission of China, and 10 provincial/regional Health Administrative Departments. The most important acknowledgement is to the participants in the study and the members of the survey teams in each of the 10 regional centers, as well as to the project development and management teams based at Beijing, Oxford and the 10 regional centers.

\section{Authors' contributions}

HW conceived and designed the study. ZC and LL, as the members of CKB steering Committee, designed the whole study. HW and MY analyzed the data and wrote the manuscript. LC, DS, YC, XZ, KX, CW, and SZ collected the data. $P P$ and $Y G$ supervised the implementation. FB provided critical comments on the manuscript and revised the manuscript. All authors read and approved the final manuscript.

\section{Funding}

This work was supported by Grants (2016YFC0900500, 2016YFC0900501, 2016YFC0900502) from the National Key Research and Development Program of China, grants from the Kadoorie Charitable Foundation in Hong Kong and Grants (212946/Z/18/Z, 202922/Z/16/Z, 104085/Z/14/Z, 088158/Z/09/Z) from Wellcome Trust in the UK. The funding sponsors have no role in design and conduct of the study; collection, analyses, and interpretation of the results.

\section{Availability of data and materials}

Details of how to access China Kadoorie Biobank data and details of the data release schedule are available from www.ckbiobank.org/site/Data+Access.

\section{Declarations}

\section{Ethics approval and consent to participate}

The CKB study was approved by the Ethical Review Committee of the Chinese Center for Disease Control and Prevention and the Oxford Tropical Research Ethics Committee, University of Oxford. All participants provided informed written consent prior to participation in the study.

\section{Consent for publication}

Not applicable.

\section{Competing interests}

The authors declare that they have no competing interests.

\section{Author details}

'Department of NCDs Control and Prevention, Zhejiang Provincial Center for Diseases Control and Prevention, \#3399 Binsheng Road, Binjiang District, Hangzhou, Zhejiang Province, China. ${ }^{2}$ Department of NCDs Control and Prevention, Tongxiang City Center for Disease Control and Prevention, Tongxiang, China. ${ }^{3}$ Chinese Academy of Medical Sciences, Beijing, China. ${ }^{4}$ Medical Research Council Population Health Research Unit, Nuffield Department of Population Health, University of Oxford, Oxford, UK. ${ }^{5}$ Department of Epidemiology and Biostatistics, School of Public Health, Peking University Health 
Science Center, Beijing, China. ${ }^{6}$ Peking University Center for Public Health and Epidemic Preparedness and Response, Beijing, China.

Received: 19 March 2021 Accepted: 4 June 2021

Published online: 06 July 2021

\section{References}

1. Lacey B, Lewington S, Clarke R, Kong XL, Chen Y, Guo Y, Yang L, Bennett D, Bragg F, Bian Z, et al. Age-specific association between blood pressure and vascular and non-vascular chronic diseases in 0.5 million adults in China: a prospective cohort study. Lancet Glob Health. 2018;6:e641-9.

2. Ettehad D, Emdin CA, Kiran A, Anderson SG, Callender T, Emberson J, Chalmers J, Rodgers A, Rahimi K. Blood pressure lowering for prevention of cardiovascular disease and death: a systematic review and meta-analysis. Lancet. 2016;387:957-67.

3. Zhou M, Wang H, Zeng X, Yin P, Zhu J, Chen W, Li X, Wang L, Wang L, Liu $Y$, et al. Mortality, morbidity, and risk factors in China and its provinces, 1990-2017: a systematic analysis for the Global Burden of Disease Study 2017. Lancet. 2019;394:1145-58.

4. Wang Z, Chen Z, Zhang L, Wang X, Hao G, Zhang Z, Shao L, Tian Y, Dong $Y$, Zheng $C$, et al. Status of hypertension in China: results from the China Hypertension Survey, 2012-2015. Circulation. 2018;137:2344-56.

5. Tapsell LC, Hemphill I, Cobiac L, Patch CS, Sullivan DR, Fenech M, Roodenrys S, Keogh JB, Clifton PM, Williams PG, et al. Health benefits of herbs and spices: the past, the present, the future. Med J Aust. 2006;185:S1-24.

6. Kaefer CM, Milner JA. The role of herbs and spices in cancer prevention. J Nutr Biochem. 2008;19:347-61.

7. Nilius B, Appendino G. Spices: the savory and beneficial science of pungency. Rev Physiol Biochem Pharmacol. 2013;164:1-76.

8. Szolcsanyi J. Forty years in capsaicin research for sensory pharmacology and physiology. Neuropeptides. 2004;38:377-84

9. Sun D, Lv J, Chen W, Li S, Guo Y, Bian Z, Yu C, Zhou H, Tan Y, Chen J, et al. Spicy food consumption is associated with adiposity measures among half a million Chinese people: the China Kadoorie Biobank study. BMC Public Health. 2014;14:1293.

10. Mei C, Fang Z, Yin R, Yang R, Tang K. Spicy food and self-reported fractures. Clin Nutr. 2019;38:2239-45.

11. Lv J, Qi L, Yu C, Yang L, Guo Y, Chen Y, Bian Z, Sun D, Du J, Ge P, et al. Consumption of spicy foods and total and cause specific mortality: population based cohort study. BMJ. 2015;351:h3942.

12. He T, Wang M, Tian Z, Zhang J, Liu Y, Zhang Y, Wang P, Xue Y. Sexdependent difference in the association between frequency of spicy food consumption and risk of hypertension in Chinese adults. Eur J Nutr. 2019:58:2449-61.

13. Xue Y, He T, Yu K, Zhao A, Zheng W, Zhang Y, Zhu B. Association between spicy food consumption and lipid profiles in adults: a nationwide population-based study. Br J Nutr. 2017;118:144-53.

14. Bonaccio M, Di Castelnuovo A, Costanzo S, Ruggiero E, De Curtis A, Persichillo M, Tabolacci C, Facchiano F, Cerletti C, Donati MB, et al. Chili Pepper Consumption and Mortality in Italian Adults. J Am Coll Cardiol. 2019:74:3139-49.

15. Shi Z, El-Obeid T, Riley M, Li M, Page A, Liu J. high chili intake and cognitive function among 4582 adults: an open cohort study over 15 years. Nutrients. 2019;11:1183.

16. Ahuja KD, Robertson IK, Geraghty DP, Ball MJ. The effect of 4-week chilli supplementation on metabolic and arterial function in humans. Eur J Clin Nutr. 2007:61:326-33.

17. Shi Z, Riley M, Brown A, Page A. Chilli intake is inversely associated with hypertension among adults. Clin Nutr ESPEN. 2018;23:67-72.
18. Harada N, Okajima K. Effects of capsaicin and isoflavone on blood pressure and serum levels of insulin-like growth factor-l in normotensive and hypertensive volunteers with alopecia. Biosci Biotechnol Biochem. 2009;73:1456-9.

19. Chen Z, Lee L, Chen J, Collins R, Wu F, Guo Y, Linksted P, Peto R. Cohort profile: the Kadoorie study of chronic disease in China (KSCDC). Int J Epidemiol. 2005;34:1243-9.

20. Chen Z, Chen J, Collins R, Guo Y, Peto R, Wu F, Li L. China Kadoorie Biobank collaborative g: China Kadoorie Biobank of 0.5 million people: survey methods, baseline characteristics and long-term follow-up. Int J Epidemiol. 2011;40:1652-66.

21. Li LM, Lv J, Guo Y, Collins R, Chen JS, Peto R, Wu F, Chen ZM. China Kadoorie Biobank Collaborative G: The China Kadoorie Biobank: related methodology and baseline characteristics of the participants. Zhonghua Liu Xing Bing Xue Za Zhi. 2012;33:249-55.

22. Liu X, Bragg F, Yang L, Kartsonaki C, Guo Y, Du H, Bian Z, Chen Y, Yu C, Lv J, et al. Smoking and smoking cessation in relation to risk of diabetes in Chinese men and women: a 9-year prospective study of 0.5 million people. Lancet Public Health. 2018;3:e167-76.

23. Im PK, Millwood IY, Guo Y, Du H, Chen Y, Bian Z, Tan Y, Guo Z, Wu S, Hua Y, et al. Patterns and trends of alcohol consumption in rural and urban areas of China: findings from the China Kadoorie Biobank. BMC Public Health. 2019;19:217.

24. Bennett DA, Du H, Bragg F, Guo Y, Wright N, Yang L, Bian Z, Chen Y, Yu C, Wang $S$, et al. Physical activity, sedentary leisure-time and risk of incident type 2 diabetes: a prospective study of 512,000 Chinese adults. BMJ Open Diabetes Res Care. 2019;7:e000835.

25. Du H, Li L, Whitlock G, Bennett D, Guo Y, Bian Z, Chen J, Sherliker P, Huang $Y$, Zhang N, et al. Patterns and socio-demographic correlates of domainspecific physical activities and their associations with adiposity in the China Kadoorie Biobank study. BMC Public Health. 2014;14:826.

26. Kanazawa M, Yoshiike N, Osaka T, Numba Y, Zimmet P, Inoue S. Criteria and classification of obesity in Japan and Asia-Oceania. World Rev Nutr Diet. 2005;94:1-12.

27. Group of China Obesity Task Force. The guideline for prevention and control of overweight and obesity in Chineses adults. Acta Nutr Sin. 2004;26:1-4.

28. Wen Q, Wei Y, Du H, Lv J, Guo Y, Bian Z, Yang L, Chen Y, Chen Y, Shi L, et al. Characteristics of spicy food consumption and its relation to lifestyle behaviours: results from 0.5 million adults. Int J Food Sci Nutr. 2021;72:569-76.

29. Yang D, Luo Z, Ma S, Wong WT, Ma L, Zhong J, He H, Zhao Z, Cao T, Yan $Z$, et al. Activation of TRPV1 by dietary capsaicin improves endotheliumdependent vasorelaxation and prevents hypertension. Cell Metab. 2010;12:130-41.

30. Ma L, Zhong J, Zhao Z, Luo Z, Ma S, Sun J, He H, Zhu T, Liu D, Zhu Z, Tepel M. Activation of TRPV1 reduces vascular lipid accumulation and attenuates atherosclerosis. Cardiovasc Res. 2011;92:504-13.

31. Zhang LL, Yan Liu D, Ma LQ, Luo ZD, Cao TB, Zhong J, Yan ZC, Wang LJ, Zhao ZG, Zhu SJ, et al. Activation of transient receptor potential vanilloid type-1 channel prevents adipogenesis and obesity. Circ Res. 2007;100:1063-70.

32. Li Q, Cui Y, Jin R, Lang H, Yu H, Sun F, He C, Ma T, Li Y, Zhou X, et al. Enjoyment of spicy flavor enhances central salty-taste perception and reduces salt intake and blood pressure. Hypertension. 2017;70:1291-9.

\section{Publisher's Note}

Springer Nature remains neutral with regard to jurisdictional claims in published maps and institutional affiliations. 